\title{
BODY AREA NETWORKS
}

\author{
Kavya Laalasa Karanam', Meghana Gotlur ${ }^{2}$ \\ ${ }^{1}$ B Tech, Computer Science and Engineering, Sree Vidyanikethan Engineering College, Andhra Pradesh, India \\ ${ }^{2} B$ Tech, Computer Science And Engineering, Sree Vidyanikethan Engineering College, Andhra Pradesh, India
}

\begin{abstract}
In recent era, miniaturized computing and sensor networks are rapidly growing in Networking. Body area network (BAN) technology has evolved in recent years as a category of wireless sensor network technology which concentrates mainly on changes in biological conditions in human beings and animals. The motivation for our work rises from the increasing health monitoring needs for the population and the self-awareness that people want to have of their physical activity. These are the main motives for the growth of sensing around and within our body. BAN has a variety of applications, including emergency response and device control, health and fitness monitoring, military and entertainment. Health and fitness monitoring includes an efficient way to continuously monitor the human body's heartbeat, blood pressure changes, sudden increase in body temperatures, increase in glucose and even brain activities through implanted/wearable sensor devices.This technology is used in many areas of human computer interaction which include brain computer interface. There are many IEEE versions related to BAN rather the current versions mainly concentrates on security and privacy. However, BAN technology also has various challenges seldom due to the scarce hardware, radio communication properties and environment where they work. BAN technology has overcome many challenges related to sensor networks. Here we will convey the need for wireless sensor networks, various applications pertaining to different fields like electrocardiography (ECG), electroencephalography (EEG) and Electromyography (EMG), Body area Network architecture, Hardware required for this technology and sensor node communications, Future scope and characteristics regarding their implementation.
\end{abstract}

Keywords: Wireless Sensor Networks, Electrocardiography, Electromyography, Electroencephalography

\section{INTODUCTION}

Body Area Networks also called as Wireless Body area networks or Body sensor Networks(BSN) is a wireless network of wearable computing devices which may be implanted inside the body or can be wore on the body. These wearable devices are sensors which sense the physiological conditions of the body. Paragraph comes content here. There are many applications in different areas like sports, Medical, Military and entertainment .This technology has overcome the drawbacks of wired sensor networks.

\subsection{Drawbacks of Wired Networks}

There are many drawbacks in wired networks. Wireless environments change dynamically, however, they are not far behind in terms of speed compared to wired networks. A wireless network speed depends generally on frequency of the signal. Higher frequency results in higher payload that the signal can transmit. Whereas wired networks, speed depends rate at which data is transmitted, the topology of network and the effectiveness of the protocol used.

In recent times everything is automated. So wireless is preferable in many places like hospitals, Schools, offices, rather than wired networks because of advantages of point to point, point to multipoint and mesh networks which are more reliable and in these dynamic re-routing of packets takes place when congestion occurs [3]. The basic problem in wired networks is that we follow connection oriented mechanism in which if congestion occurs at appoint it is difficult to redirect the route of the packets. Since we follow the same route to transfer the packets if a security attack takes place in that route then eavesdropping can be done. Installation and Maintenance of wires is to be done carefully from source to destination unlike wireless which is simple and easy to maintain. Wireless networks is cost effective.

\subsection{IEEE DEFINITION OF BAN}

A communication standard used by devices operating in and around human body and animals for various applications comprising medical, electronic devices and entertainment.

\section{BAN ARCHITECTURE}

The layered architecture is similar to TCP/IP reference model. There are four layers as depicted in the diagram.

\subsection{Physical Layer}

The lowest and basic layer in a BAN's communication stack is PHY, which is responsible for transmitting raw information bits by a transmitter and receiving these information bits by receiver. Many frequency bands are used for communications between nodes around the human body The frequency ranges around $403 \mathrm{MHz}$ is suitable for implanted sensors in medical applications , $13.5 \mathrm{MHz}, 400$ $\mathrm{MHz}, 900 \mathrm{MHz}, 2.4 \mathrm{GHz}, 3.1-10.6 \mathrm{GHz}$ suitable for onbody sensors. In general, radio signal most of the time diffracts out of the body rather than to pass through the human body. This will become a problem if the sensors are implanted on two opposite directions in the human body resulting in information loss. 


\subsection{Medium Access Control Layer}

In a BAN, the nodes transmit through a shared channel. Accessing the shared channel is monitored by a MAC Layer present in every sensor node of BAN. MAC protocols can be categorized as: schedule-based and contention-based. Schedule-based MAC protocols follow the principle that all nodes can equally access shared channel. Packet collision is avoided since the nodes can access shared channel according to different time slots allotted to them. Contention-based MAC protocols follow the principle that nodes decides when and how the channel is accessed. Contention-based MAC protocols are more realistic and practically applicable in this BAN because of their scalability. Energy-efficient MAC protocols can be employed since they play a vital role in decreasing energy consumed thereby increasing network's lifetime. Quite a few MAC Protocols have been suggested including Controlling Access with Distributed slot Assignment (CICADA) aims to concentrate mainly on BAN with huge traffic, BAN-MAC for STAR Topology BANS and for best energy conservation. Hybrid MAC (H-MAC) protocol to improve energy efficiency for bio-sensors and provide time synchronization [6].

\subsection{Network Layer}

Efficient packet transmission and finding the optimal route from one node to other is the role of a network layer protocol. Routing protocols maintained in Zig Bee's network layer are the Ad hoc On-demand Distance Vector (AODV) protocol which does routing operations when required and the other protocol is Hierarchical Routing Algorithm (HERA), which depends hierarchical structure during network construction. Various protocols used are Sensor Protocols for Information via Negotiation (SPIN) and Threshold sensitive Energy Efficient sensor Network (TEEN).

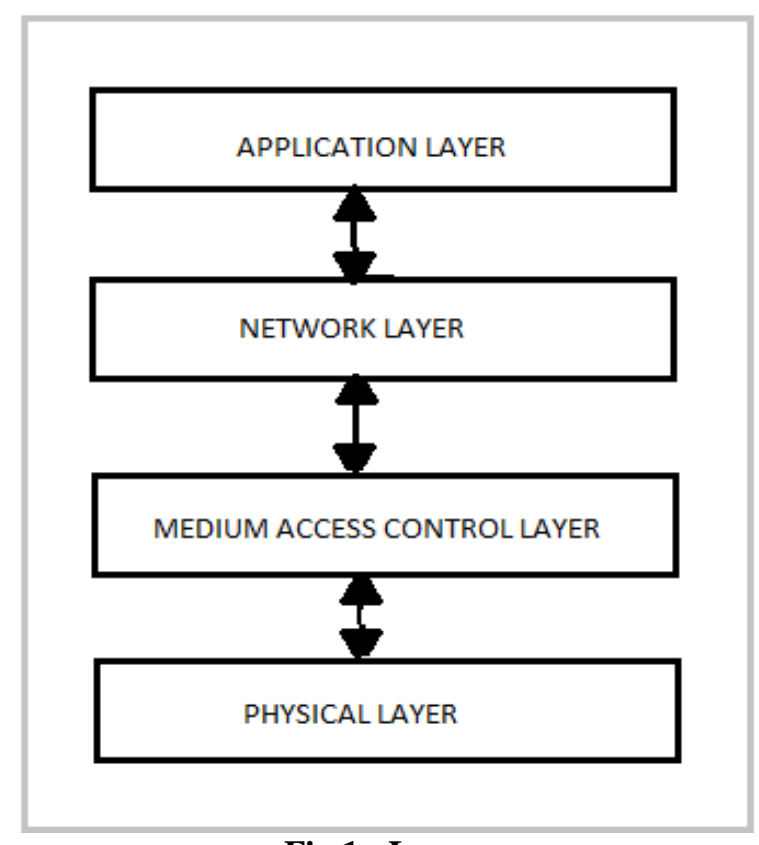

Fig 1 : Layers

\subsection{Application Layer}

The important component of this application layer is Operating Systems. The Operating System be able to manage the radios, sensors, timers, memory while providing an abstraction of application programming interfaces (APIs). The application interface changes for various applications of BAN. The Operating system for a BAN is lightweight and simple since sensor nodes have constraints on resources used like computational power.

\subsection{Basic Application Architecture}

The architecture in the diagram shows that whenever patients embedded or wore sensors detect some problems it signals the personal server first level [6]. This information is then transferred to any nearby system through WLAN or GPRS in second level. This information is further sent through internet to patient's friends, relatives and to patients doctor immediately.

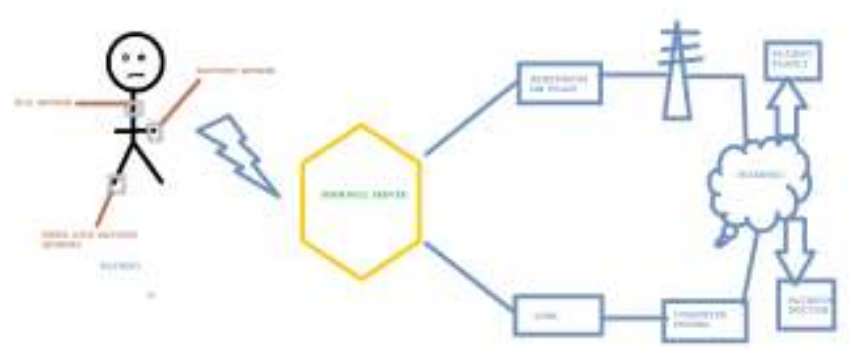

Fig 2 : 3 Tier Architecture

\section{Associated Technologies}

Supporting Technologies include ZigBee, IEEE Standard for Local and metropolitan area networks (IEEE 802.15.4 ) and basic Bluetooth application. The IEEE 802.15.4 covers physical and MAC layers mainly focusing on low rate short distance radio communications among nodes. The ZigBee improves the IEEE 802.15.4 Standard by increasing security and frameworks to facilitate a larger Wireless Sensor Networks (WSN).

\section{Applications}

There are wide range of applications for this Ban Technology which includes Medical applications, Entertainment, Fitness, Sports and Military. The increase in fast computing devices, Sensors and miniaturization of these devices has led to these wide range of applications of daily routine.

\subsection{Medical Applications}

The Population is increasing day by day and it is expected by 2030 there will be more than 750 million old people around the world. So the older people are there will be greater need for different ways to monitor their medical status and stay at home monitoring their health condition without staying at hospital. Some of the physiological sensors used in WBAN is ECG (Electrocardiogram) Sensor which monitors the heart rate or the hearts activity 
continuously, EMG (Electromyography) sensor which monitors the electrical signals generated by the muscles continuously, EEG (Electroencephalography) sensor which monitors the electrical activity of the brain which is basis for brain computer interaction [1]. Other sensors include for measuring blood pressure and breathing sensors.

\subsubsection{Electrocardiogram (ECG)}

Heart is a nutrient rich organ that pumps blood throughout the body. Each heart beat is stimulated by the electrical signal that pass through heart muscle or myocardium .In order to check the function of heart a doctor may perform the ECG. It is method of observing the electrical activity of the heart for some time by placing electrodes on a patient's body. These electrodes detect the tiny electrical changes on the skin that arise from the heart muscle depolarizing during each heartbeat. Generally the Electrocardiogram generated, which if it is abnormal leading to deadly diseases like Heart attack. With the help of these sensors, placed, the abnormality in electrical activity is indicated at the earlier stage which can lead to prevention of heart attack by adopting certain measures [2]. With the help of these sensor, one can detect heart attack prior to the attack.

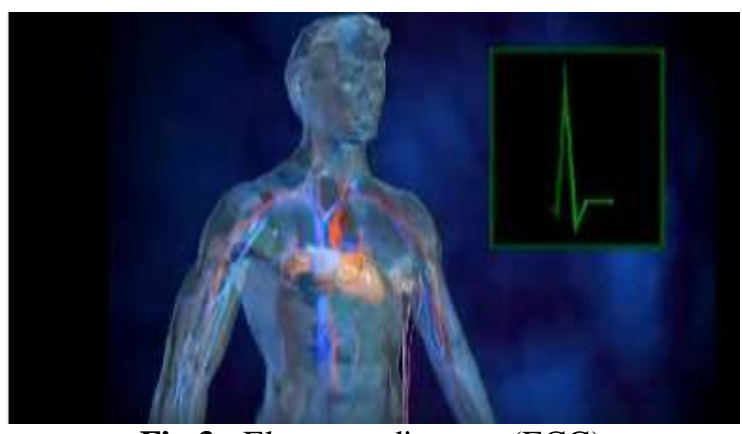

Fig 3 : Electrocardiogram (ECG)

\subsubsection{Electromyography (EMG)}

Electromyography (EMG) is a method for recording the electrical activity formed by muscles. This detects the electrical potential produced by muscle cells when they are neurologically or electrically stimulated. These signals can be used to detect any irregularities or abnormal behavior and to understand basic human movement. It helps in detecting neuromuscular diseases.

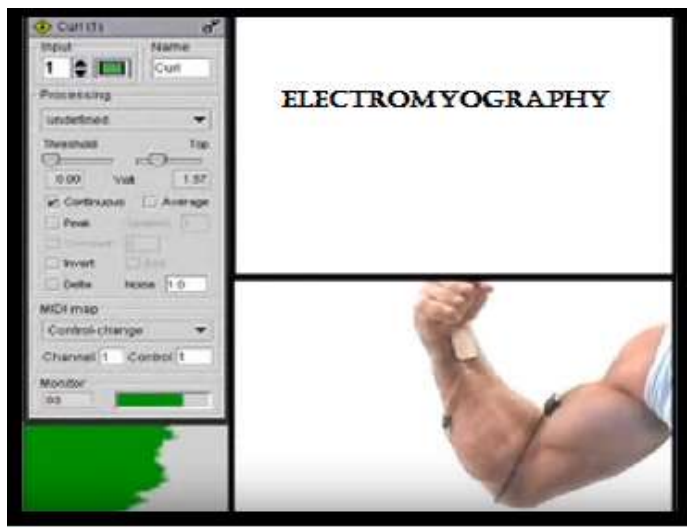

Fig 4: Electromyography (EMG)

\subsubsection{Electroencephalography (EEG)}

The basis for this application is brain computer interface. This brain computer interface is the system that allows us to interact with the computer based on the changes in electrical signals that occur in our brain .This can achieved with the help of these sensors. The electrical signals are present at the micro levels on the scalp of the brain, so these have to be amplified in order to reach to the computer. This can be amplified with this sensor and further should be digitalized by passing these signals via an analog to digital convertor (ADC) .This resulting signal is further sent to computer for analysis. These signals are analyzed by the spectrum based on amplitude and frequency and finally implementing these parameters into a neural network [8].

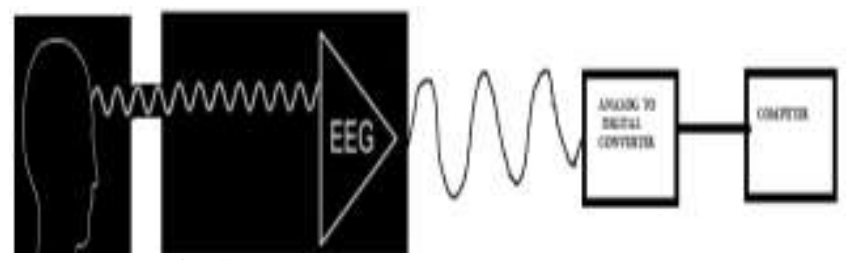

Fig 5 : Electroencephalography (EEG)

\subsubsection{Cancer Detection}

Cancer is the threat to human beings. The detection of cancer can be done using sensors which can be implanted inside the body such that it detects $\mathrm{NO}$ (Nitric oxide) which is emitted by cancer cell. In addition these special sensors have the ability to distinguish between cancer cells and other cells of the body. Thus Ban Technology helps in Detection of cancer at the early stage which on treatment could save a persons life.

\subsubsection{Artificial Retina}

With The Help of This BAN technology, Blind people can be able to see this colorful world around them. These can see at a sensible level by implanting retina prosthesis chips inside eye, as shown in Figure.[11]

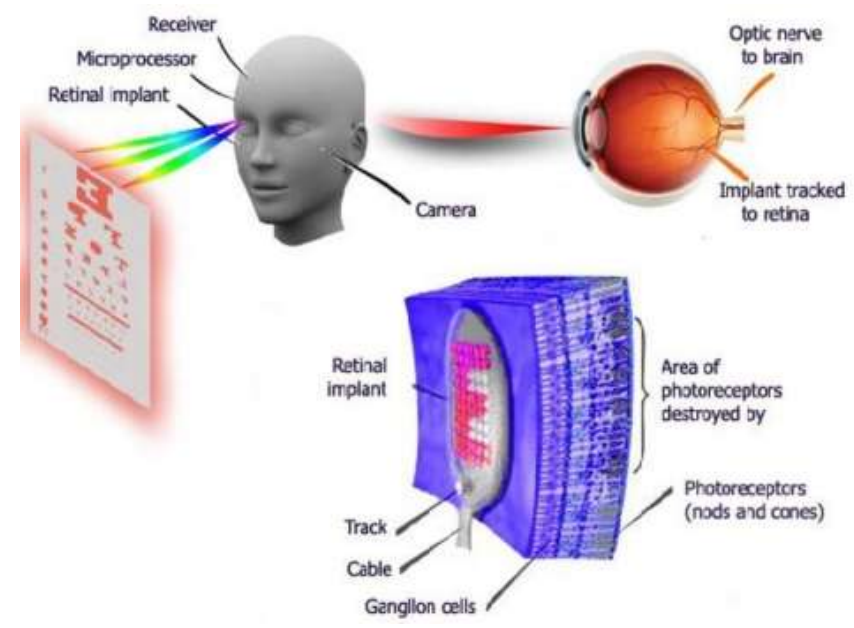

Fig 6: Non-natural Retina for Blind People 


\subsection{Sports Applications}

Among many applications of BAN in various fields we also have several applications in sports field. The major use is to detect whether a posture of body may lead to any sprain or cause any fractures. The sensors located at various joints in the body always check whether position is appropriate or not. If not then the person is immediately notified to change his/her posture. In this way sportsmen use this while playing important matches to avoid sprain and cramps.

\section{BAN Hardware}

The main hardware components of BAN are sensors, Main Control Unit and Radio transmitter. Among all these, sensors form the major component. They are particularly used to detect any physical or physiological changes that may occur in the body [7]. We have different types of sensors whose working is mentioned as follows.

Inertial motion sensors. In this we use Accelerometers and Gyroscopes .Accelerometers are used to measure gravitational pull and inclination. Gyroscopes are used to measure angular displacement. Based on these measurements we calculate and get posture of the body. This makes it useful in sports in applications where we can avoid fractures and muscular disorders.

Bioelectrical sensors. These sensors are applied to measure the electrical variations on person's skin. Generally we get some electrical variations on a person's skin based on the working condition of the internal body organ. This is mostly applied in Electrocardiography (ECG) and Electromyography (EMG).Let us how it takes place. In Electrocardiography we place circular pads on the skin of a person near the chest and these sensors measure the electrical variations. If there are any abnormal changes that we find then we can say that the person has some problem in his heart. The same thing happens in Electromyography. To diagnose neuromuscular disorders we measure the electrical activity of skeletal muscles.

Electrochemical sensors. We place sensors all over the body. These sensors have chemical agents present in them which can react with substances present in the body. The output that will be generated when this happens is in the form of electrical signals. Based on this output we can detect whether any bodily substances are generated in bulk quantities or whether there are any abnormal changes in the chemical properties of bodily substances. The best example that we can quote here is the amount of glucose in the blood. Here we employ Bloodglucose sensors and based on the electrical output generated we can estimate the amount of glucose in the blood. We also use this to measure the amount of carbon dioxide in Respiratory system and Bile juice generated in the liver to diagnose Jaundice.

Optical sensors. When the light is made to pass through arteries based on the amount of light emitted we can calculate oxygen content in blood. Here we employ devices which emit and receive light both in visible and infrared spectrum. We employ a device called Pulse oximeter to measure oxygen content. The Pulse oximeter takes the amount of light emitted and the total amount of light passed through a person's arteries and gives the oxygen content.
Temperature sensors. Here the name itself says that this sensor is used to measure the temperature of the body. We place this at different locations on the skin of person. Their job is to measure the temperature and transmit the information to Main Control Unit during fever conditions.

\section{1 .Sensor Node Communication}

We have different types of sensors placed at different locations in our body. Each senor type has a main sensor which controls all the other sensors in that particular body area. All these sensors sense and gather information. This information is sent to the Main Control Unit (MCU) . Iwhich processes the arrived information. The MCU sends this information to radio transmitter which in turn transmits the information via internet to the patient's family doctor, neighbors, family members etc. In this way communication takes place between one sensors to another sensor. If the sensors detect any abnormal changes in the physiological conditions of the body then the alarm which is present at the MCU will ring which informs emergency conditions.

\section{CHALLENGES}

This is a useful technology but still we do have certain challenges concerning BAN. Some of the challenges are given below.

Security: Security is a high priority in all networks. Therefore considerable effort is necessary to make BAN a secure network. It is necessary to ensure that the data of a particular patient is not mixed up with other patient's data over the network. We should also provide access rights to this data i.e. they should be accessible to patient, doctor and few family members. Others are not allowed to access this data. The data should be confidential, authorized and access rights must be provided to the system and the data should not be modified when transmission is taking place over the network. The IEEE 802.15.16 is latest standard which has features to provide security in WBAN [4]. But still there are many problems related security aspects. Research is further going on in this field

Interoperability: WBAN systems should ensure that data transfer is continuous and there shouldn't be any interruptions over the network. In this the data generated is high because we employ more hardware devices. Therefore the output generated is also in terms of the same quantity. So we should make sure that the system is scalable i.e. it should have the capacity to handle the large amount of data generated.

System devices: The sensors in BAN should be less complicated. Since the sensors are put on the human body they should be light in weight, easily reconfigurable, power efficient and easy to use. The user should not feel uncomfortable wearing these devices.

Sensor validation: Sensor devices have several hardware constraints which include unreliable wired/wireless network links, interference between the networks and limited power supply. This results in generation of erroneous data which is transmitted to users and doctor. This leads to false alarm generation and a person who is healthy may be considered as a patient .Therefore the sensors employed in healthcare 
domain should produce valid results weaknesses in hardware and software design must be eliminated.

Data consistency: The storage devices should be located in remote locations and consistent viewing of patient data and analysis is to be done. The data should be resided on patient's family doctor's PC or mobile phone. If it does not contain entire data then the Quality of treatment is degraded. Cost: Now-a-days people expect low cost health monitoring systems with high functionality. We can see that this is a highly productive device but since we employ sensors it is very expensive. We should make this cost effective and see that it available to common man.

Constant monitoring: Constant monitoring of patients is done and it is different for different users. This difference is based on the kind of health problem they have. For example if the user is a heart patient then constant monitoring of heart is essential whereas for users who have risk of falling needs monitoring when they are walking or running. If this fails then it may cause serious effects on the life of the patient.

Constrained deployment: The WBAN should be wearable, light in weight and should not cause any disruption or annoyance. It should not disturb the user's daily activities. The system should monitor in such a way that user does not realize anything.

Consistent performance: The performance should be consistent. Sensor measurements should be accurate and the system should save the values, even when the WBAN is switched off and switched on again. The abnormal conditions of the body should be recognizedaccurately by the sensors. The working and generation of results should be faster in to avoid emergency conditions. The wireless links should be robust and work under extreme conditions [9].

\section{CONCLUSIONS}

The future market of WBAN is growing rapidly in the field of healthcare and in the entertainment industry .The WBAN systems will allow a dramatic shift in the way people think about and manage their health. In todays busy we don't find time for regular medical diagnosis. Since this system does monitoring constantly and does not need human intervention Quality of Life is improved. Not only has that it reduced the healthcare costs. This has the potential to offer a wide range of benefits to patients, medical personnel and society through early detection of possible problems. By detecting abnormal changes in the body we can avoid emergency conditions like heart attacks and cancer. This not only has applications in medical field but also in Brain Computer Interface.

\section{REFERENCES}

[1].Wireless Body Area Network https://www.youtube.com/watch?v=7uE7_LBliQo

[2].WBAN -Telemetry

https://www.youtube.com/watch?v=Jgv6cF7CQGs

Robot

[3].Comparing wireless sensor networks - wired vs wireless http://www.rcrwireless.com/20120731/opinion/realitycheck-comparing-wireless-sensor-networks-wired-vswireless
[4]. https://en.wikipedia.org/wiki/Body_area_network [5]. Milenkovic A, Otto C, Jovanov E (2006) Wireless sensor networks for personal health Monitoring: issues and an implementation. Compute Commun 29(13-14):25212533

[6]. IEEE Standard 802, Part 15.4: Wireless Medium Access Control (MAC) and Physical Layer

(PHY) specifications for low-rate wireless personal area networks (WPANs), IEEE Std Body Area Networks 35

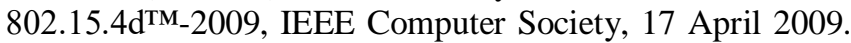
Available(2012):http://standards.ieee.org/about/get/802/802. 15.html.

[7]. Jurik AD, Weaver AC (2009) Body sensors: wireless access to physiological data. IEEE Softw26 (1):71-73

[8] Medical applications of BAN http://www.aicit.org/jdcta/ppl/23.pdf

[9] Futuristic issues and challenges of BANhttp://ijcsi.org/papers/IJCSI-9-1-2-180-186.pdf

[10] National Center for Health Statistics, URL: http://www.cdc.gov/nchs/Default.htm, Accessed in June 2009.

[11] L. Schwiebert, S. k. S. Gupta, and J. Weinmann, "Research Challenges in Wireless Networks of Biomedical Sensors," in 7th annual International Conference on Mobile Computing and Networking, Rome, Italy, 2001, pp. 151165.

\section{BIOGRAPHIES}

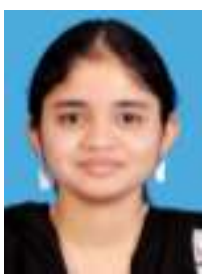

I am Meghana Gotlur. Presently I am pursuing my graduation in Computer Science and Technology in Sree Vidyanikethan Engineering College, Tirupati.

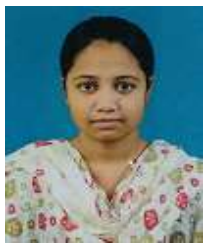

I am Kavya Laalasa Karanam. Presently I am pursuing my graduation in Computer Science and Technology in Sree Vidyanikethan Engineering College, Tirupati. 